\title{
Beam Emittance and the Effects of the RF, Space Charge and Wake Fields - Application to the ATF Photoelectron Beam
}

\author{
Zohreh Parsa \\ Brookhaven National Laboratory \\ Upton, NY 11973
}

\begin{abstract}
Laser driven photelectron guns are of interest for use in new methods of accelerations, future development of Linear Colliders and new experiments such as Free Electron Laser (FEL) and Inverse Free Electron Laser (IFEL). Such guns are potential source of low emittance - high current and short bunch length electron beams, where the emitted electrons are accelerated quickly to a relativistic energy by a strong RF, electric field in the cavity. We present a brief overview of the beam dynamic studies, e.g. emittance for the Brookhaven National Laboratory (BNL) ATF high brightness photocathode radio frequency gun (now in operation), and show the effects of the RF. Space Charge, and Wake fields on the photoelectrons.
\end{abstract}

\section{INTRODUCTION}

This presentation includes beam dynamic analysis (e.g. calculations of emittance, energy, etc), with the effects of the RF, Self - field and Wake field on the particles in a cavity, e.g. photoelectrons, in the BNL photoelectron gun cavity. In that we show the effects of the interactions between the charged particles and the RF, Space Charge and the Wake fields on the beam dynamics. In section III, we give a sketch of the formulation used. (For more detailed analysis of the Gun e.g. see the Refs.)

\section{BNL PHOTOCATHODE GUN:}

BNL Gun consists of a $11 / 2$ cell, pi mode, resonant disk loaded structure (with the structure inner diameter of $83.03 \mathrm{~mm}$, and length of $78.75 \mathrm{~mm}$, operating at $2856 \mathrm{MHz}$ ), used as the injector to the $50 \mathrm{MeV}$ electron LINAC at BNL Accelerator Test Facility (ATF). Designed to produce electron bunches which can be synchronized with the pico second (ps) pulse of 100 - Gwatt CO2 laser. The beam brightness is maximized by placing the photocathode at the end wall of the cavity that supports a strong standing wave field; the electric fi: gradients on the cathode is $100 \mathrm{MV} / \mathrm{m}$.

Figure 1, shows a sketch of the BNL photocathode gun.

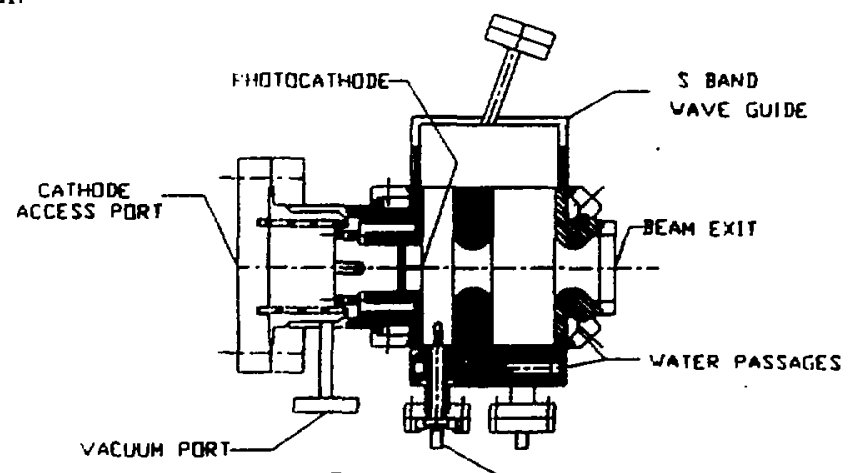

Figure $1 \longrightarrow$ RF TUNER

Figure 2 a,b,c, shows variations in the normalized transverse, longitudinal emittance, and kinetic energy (and momentum), with respect to the variations in the initial phase angle. From these we sote that the lowest emittance (and the highest momentum) occurs at the initial phase of $\sim 55^{\circ}$

Thus in the following figures we illustrate the effects of the self- field, and wake field at various points along the cavity for the "standard" BNL gun, with $100 \mathrm{11} / \mathrm{m}$ field gradient on the cathode, the laser spot size of $3 \mathrm{~mm}$. and the laser pulse width of 2 ps, (i.e. $6 \mathrm{~mm}$ ). For clarity. we note the following definition used in this analysis; 10 malized Transverse Emittance $=\sqrt{x^{2} p_{x}^{2}-\left(x p_{i}\right)^{2}}$. R.MS Transverse Emittance $=\sqrt{x^{2} x^{\prime 2}-\left(x x^{\prime 2}\right)}$, (for discussion of emittance including higher order moments see $[3]$ ).

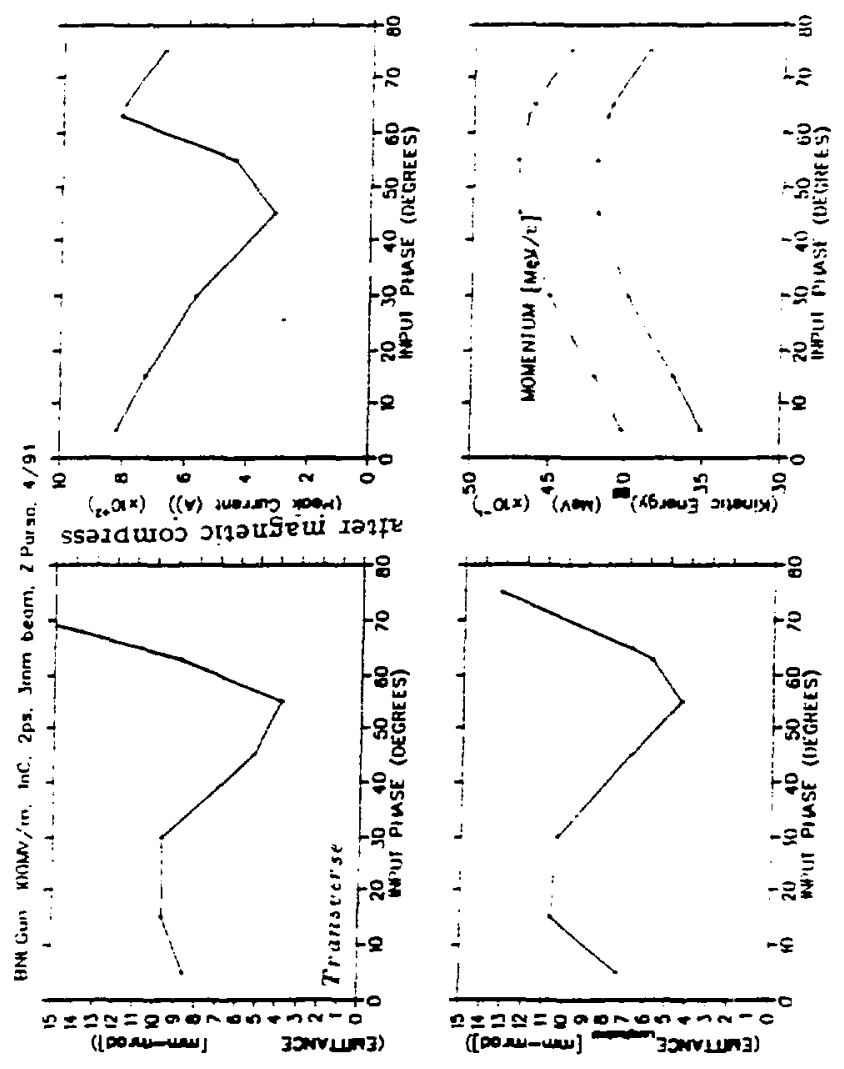

Figure 2

The kinetic energy of the particles on the cathode surface is $0.2 \mathrm{eV}$, the normalized transverse emittance is $1.28 \mathrm{~mm}$-mrad. The particles are distributed uniformly in radial divergence and their charge follow a Maxwellian distribution in radius in order to get a gaussian distribution for the bunch charge density. The $\sigma_{R}$ of the distribution used is $3 \mathrm{~mm}$ thus the RMS radius $=\sqrt{2} \times \sigma_{R}$ is $\sim 4.2 \mathrm{~mm}$, with the Max Divergence $=1.561 \mathrm{rad}$. and lie R.MS Divergence of 0.904 rad. 
Figures 3 a-e show the constant field lines and the positions of the bunch at which points, the interaction between the bunch self field and the wake-field induced in the cavity (by the bunch as it passes through the cavity) are shown in Figure 4. Figure $3 \mathrm{e}$, shows the position of the bunch at $z \sim 98 \mathrm{~mm}$, where the normalized transverse emittance $\epsilon_{i}^{V}=\tau .2 \mathrm{~mm}$-mrad, the Average kinetic energy is $4.2 \mathrm{MeV}$. Additional information on the beam dysnamics of the bunch at this position are shown in Figures $5-\bar{i}$. Figure 5 , shows the deriving current density $J_{z}$ (produced by the bunch shown in Fig. 3e), and the field as functions of $z$. Figure 6 , shows the beam emittance, energy, and the rms bunch length as functions of $z$, and Fig. 7 shows the phase space plot of $R$ vs $P_{R}$.

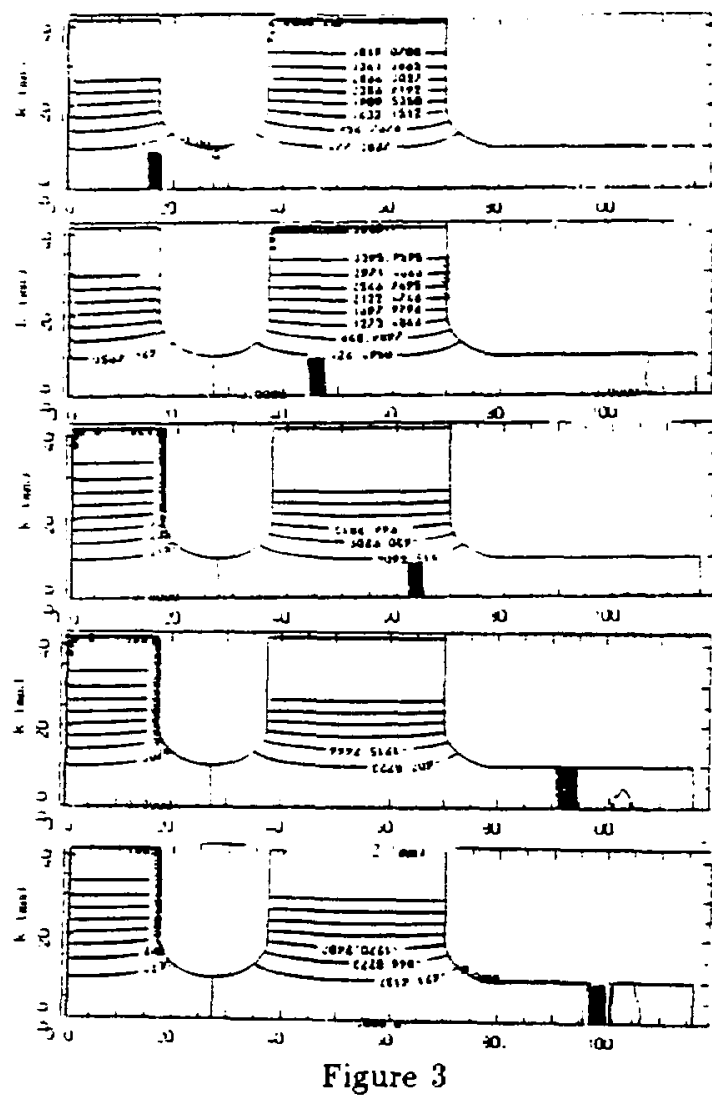

\section{FORMALISM}

This section provides a brief (due to space limitations) sketch of the formalism used to study the interaction of charged particle bunches with the fields inside the acelerating structures, (e.g. the BNL photocathode gun discussed in section II).

We assumed that the fields $\vec{E}=E(r, z, t)$ and $\vec{H}=$ $H(r, z, t)$; and the source $J=J(r, z, t)$, and $\rho=\rho(r, z, t)$ are axisymmetrical, hence the wave equations can be rexpressed as two uncoupled sets of $T M$-like mode $\left(H_{\phi}\right.$, $\left.E_{r}, E_{z}\right)$ and $T E$-like mode equations.

Since a general $T M$ field can be expanded as a sum of infinite $T M_{\circ \text { onp }}$ modes of a resonant cavity; and a general $T E$ field can be expanded as a sum $c$ f infinite $T E_{\text {onp }}$ modes of a resonance cavity ( with $J_{r}$ and $J_{z}$ as the driving currents for the $T . M$ field and $J_{\phi}$ as the driving current for the $T E$ field), a charged particle $q$ moving with a velocity $\vec{v}=v_{r} \vec{e}_{r}+v_{z} \vec{k}$ inside a $T M$ field is

$3 \mathrm{~d}$

$3 e$ subject to a force $\vec{F}=F_{r} e_{r}+F_{z} \vec{k}$ and gives rase 10 :a current $J=J_{r} \vec{e}_{r}+J_{z} \vec{k}$. When the particie is anside a $T E$ field, $\mathrm{i}_{2}$ is subject to a force $\vec{F}=F_{0} \vec{e}_{0}$ that guves rise to a current $\vec{J}=J_{r} \vec{e}_{r}+J_{\theta} \vec{e}_{\theta}+J_{z} \vec{k}$. Then for an umtua: driving current of the type; $\vec{J}=\left(J_{\tau}\left(r_{.} z . t\right), J_{z}\left(r_{.} z t\right)\right) \mathrm{in}$ presence of a $T M$ field,
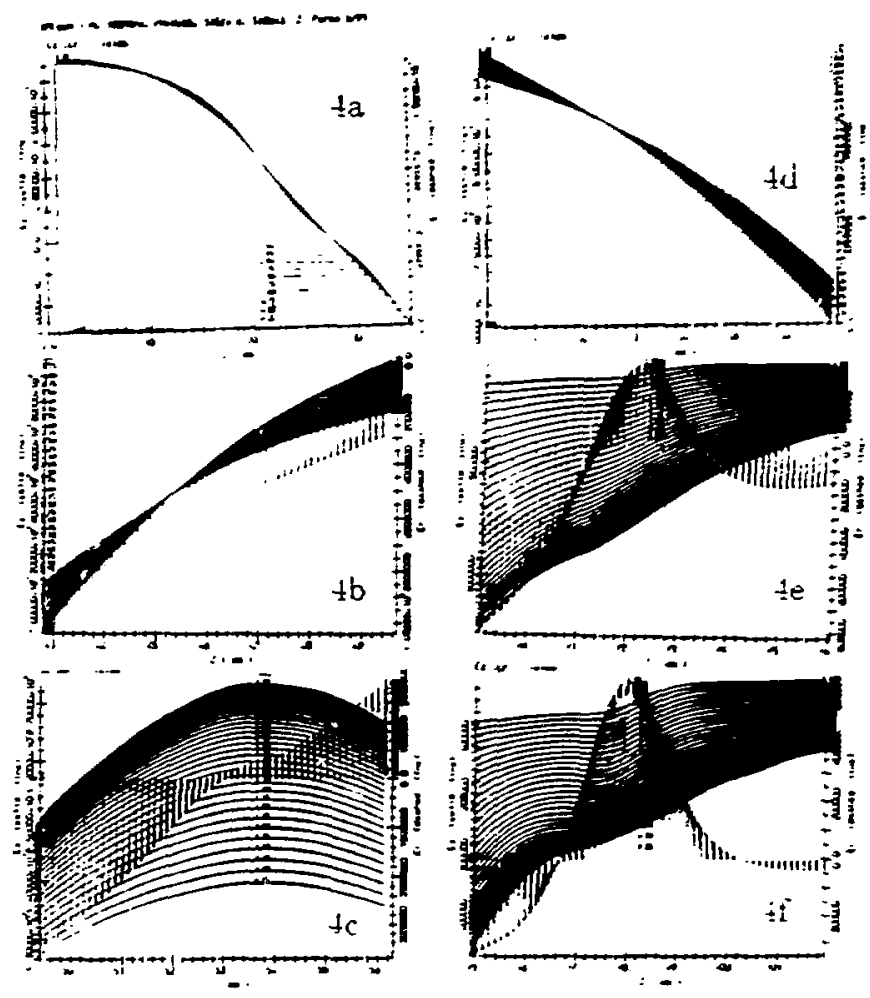

Figure 4

and

$$
\nabla^{2} \vec{H}-\frac{1}{c^{2}} \frac{\partial^{2} \vec{H}}{\partial t^{2}}=-\nabla \times \vec{J}
$$

$$
\frac{d \vec{p}}{d t}=q(\vec{E}+\vec{v} \times \vec{B})
$$

form a set of "self-consistent" system of equations. Wher" $\vec{v}=\left(v_{r}, v_{z}\right)$. thus the beam-cavity interaction can be completely described, from the point of view of the field propagations, by means of one scalar equation:

$$
L \phi=\frac{1}{c^{2}} \frac{\partial^{2} \phi}{\partial t^{2}}-r \frac{\partial J_{\Sigma}}{\partial r}-r \frac{\partial J_{r}}{\partial z}
$$

This hyperbolic equation gives the time evolution of the fields from some initial time $t=0$ (knowing the driving current) where $L \equiv \frac{\partial^{2}}{\partial z^{2}}+\frac{\partial^{2}}{\partial r^{2}}-\frac{1}{r} \frac{\partial}{\partial r}$, and the pseudo scalar potential $\phi=r H_{\phi} ; H_{\phi}=H_{\phi}(r, z, t), J_{r}=J_{r}(r, z, t)$ and $J_{z}=J_{z}(r, z, t)$. The driving current is due to the motion of the bunch of particles around the axis of cavity. and since the deriving current must be axisymmetrical the particles (of the bunch) can only move in the $r$ - z plaue (so their charge density and current are the same as lliose? produced by "rings of charge" centered on - axis): ian move axially or expand radially.

Using the standard Finite Difference Method I FDM, that allows the transform of the differential problems mio 
the algebraic ones, via the discretization of the operators $(L)$ and functions $\left(0, J_{r}, J_{z}\right)$, over a mesh, one solves simultaneously the equations, for the field propagation and the equation of motion for the particles. Thus the effects of the e.m. interactions on the beam dynamics properties, such as emittances. energy spreads, ...can be oblained (e.g. with the code ITACA), knowing the field $H_{\theta}$ at each mesh point at present and at next integration time the equation is integrated with respect to the standard $R_{\text {. }} K$. methods

$$
\begin{aligned}
& \frac{\partial E_{r}}{\partial r}=-\frac{\partial H_{\phi}}{\partial z}-J_{r} \\
& \frac{\partial E_{z}}{\partial \tau}=\frac{1}{r} \frac{\partial\left(r H_{\phi}\right)}{\partial r}-J_{z}
\end{aligned}
$$

where the charge distribution obtained becomes:

$$
\begin{aligned}
p_{i}\left(r, r_{i}, z_{2} z_{i}\right) & =e^{-\frac{\left(i-i_{1}\right)^{2}}{2 \sigma_{i}^{2}}-\frac{\left(r-r_{1}\right)^{2}}{2 \sigma^{2}}} e^{-\left(\frac{r_{r}}{\sigma_{r}^{2}}\right) \alpha_{0 i}} \\
\alpha_{n_{i}} & =\frac{q_{i}}{(2 \pi)^{3 / 2} \sigma_{r}^{2} \sigma_{z}} \bullet I_{0}\left(\frac{r r_{i}}{\sigma_{r}^{2}}\right)
\end{aligned}
$$

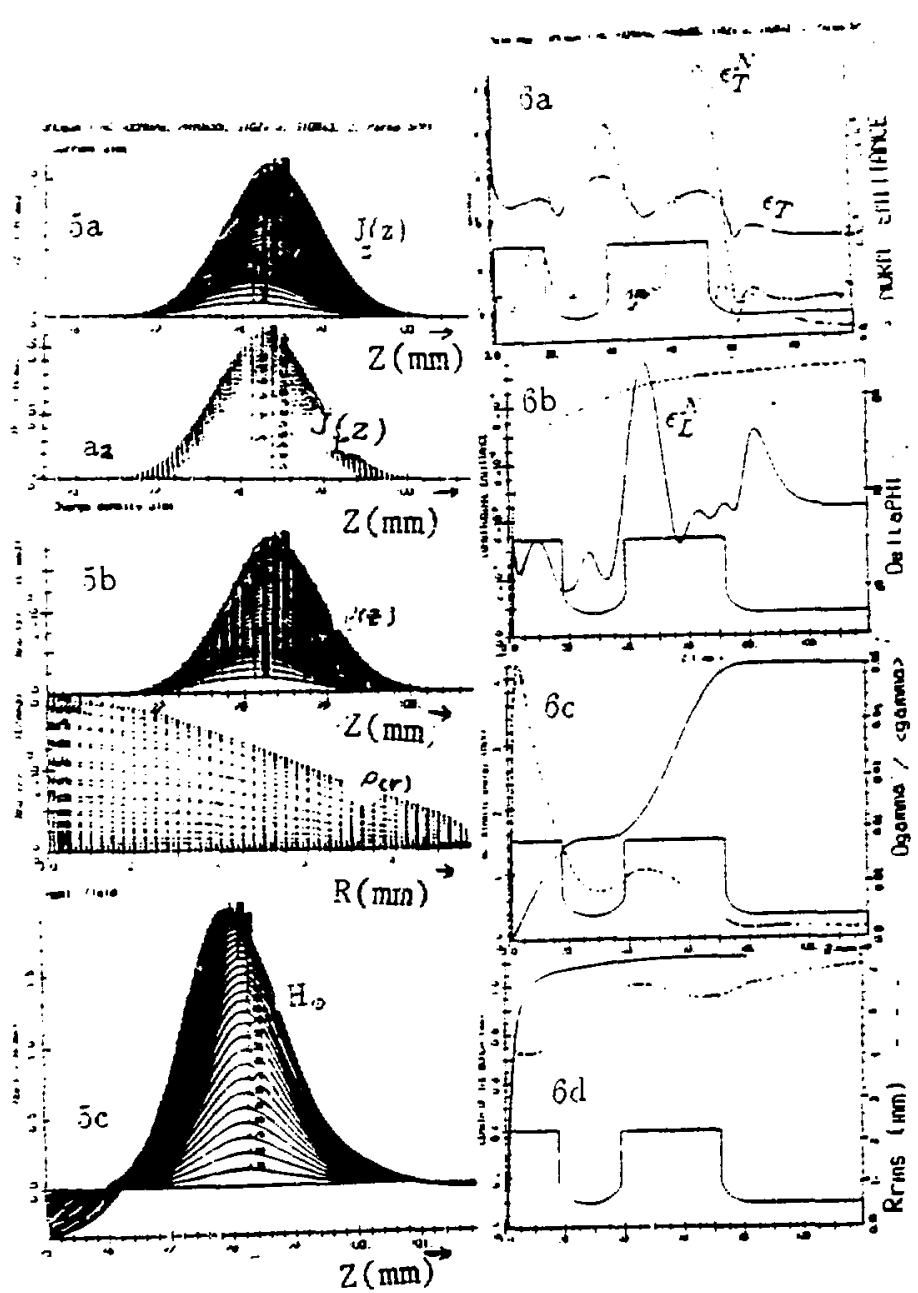

Figure 5 and the corresponding curren 2 densities becone

$$
\begin{aligned}
& J_{z}=3_{\|}^{i} \alpha_{0 i} e^{-\left(i z-z_{0}\right)^{2} / 2 \sigma_{i}^{2}} e^{\left(-r^{2}-r_{i}^{2}\right) / 2 r^{2}}
\end{aligned}
$$

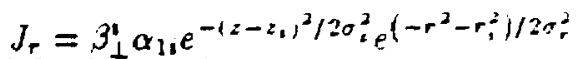

which satisfy the continuity equation. guarantees gasige invariance $(\nabla \cdot \vec{E}-\rho / \varepsilon=0)$ and minimizes the unphysuai fluctuation in the driving current.

Where, in Eqs. (5-7) $q, \sigma_{r}, \sigma_{z}$ are the charge and coordinates of the $i$-th particle; $\beta_{\perp i}$ and $\beta_{i, j}$ are the radial and axial components of the $i$-th particle beta resperctively; $I_{0}$ and $I_{1}$ are the 0 -th and 1 st-order modified Besse!

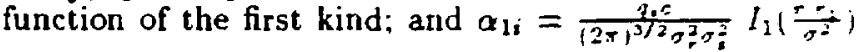
(For detailed formulation see references.)

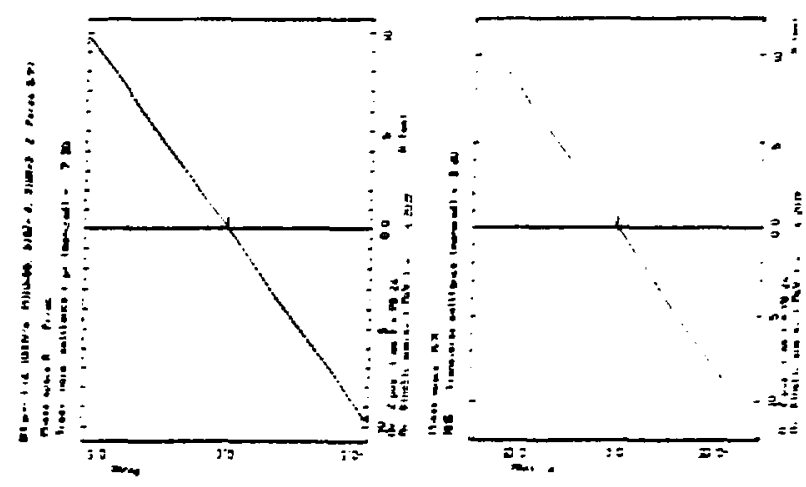

Figure 7

SUMMARY: We presented some of the results of unr studies for the BNL RF Gun. laser induced photoelectron: and showed the interaction between the self field and the wake induced in the cavity. we also presented the beam parameters obtained at the gun exit $(z \sim 98 \mathrm{~mm})$, and provided an overview of the formalism used. With this methode our preliminary analysis indicate (Fig 2), that at $100 \mathrm{MV} / \mathrm{m}$ field on the cathode and $\operatorname{lnC}$ charge the initial phase of $55^{\circ}$ will provide smallest emittance and largest Kinetic energy at $t / 1$ e gun exit. Due to space limitation, additional information is given in References.

\section{REFERENCES}

1. 2. Parsa, Preliminary emiltance measurements and calculations for the laser excited beam from BNL ATF RF gun. Proc. of BNL CAP meetings (1991) and Ref. therein: K. Batchelor, K. McDonald, et. al., Proc. of EPAC $8 \&$.

2. Private Communication, Code ITACA, L. Serafini.

3. Z. Parsa, EPAC 1990; and Ref. therein.

4. Z. Parsa, L. Young, Proc. of FEL Conf. (1991).

- We thank Fern Simes and Jerry Gaeta for assisting with the typing and printing and L. Serafini. L. Young and $K$. Batchelor for discussions. 